JEFAS

24,47

\section{2}

Received 28 June 2018 Revised 28 June 2018 Accepted 26 September 2018

\title{
Co-authorship networks of Argentine economists
}

\author{
Juan M.C. Larrosa
}

Universidad Nacional del Sur, Bahia Blanca, Argentina and Instituto de Investigaciones Económicas y Sociales del Sur (IIESS), Altos de Palihue Bahía Blanca, Argentina

\begin{abstract}
Purpose - This paper aims to provide information about the structure of collaborative work among Argentinian economics. The study provides specific applied research of social network analysis focus on this profession in this specific country.

Design/methodology/approach - The contribution opted for applying social network analysis tools to papers presented in a congress and published in its proceedings. The authors focus in detecting main actors, groups of co-authorship, professionals acting as bridges between groups and differences between genders.

Findings - The paper provides empirical insights about how co-authorship has evolved between Argentine economists. The authors find that structural properties of the network, main actors, both male and female, main universities or center that affiliates them, a gender gap that might be closing out.

Research limitations/implications - The paper focuses on the network for the period 1964-2014 without a more detailed dynamic. It also does not explain main topics worked by the authors.

Practical implications - The work provides knowledge about how groups are created in Economics in Argentina, how cooperation has evolved and what has been the role of women in this development. It also shows how different departments and entities collaborate with diverse success in the creation of new knowledge in Economics in Argentina.
\end{abstract}

Originality/value - The paper works with data from a source of information non-previously studied and contributes in explaining a particular type of collaborative work in a profession in Argentina.

Keywords Social network analysis, Community detection, Co-authorship, Economic profession, Proceedings

Paper type Research paper

\section{Introduction}

Networks of collaboration among researchers have been present since early times of science. To coauthor a paper means collaboration between at least two authors and is a way of learning, sharing knowledge and labor division. It is a common practice in scientific production and has been steadily increasing in all branches of science (De Stefano et al., 2009). These networks are affiliation networks in nature (such as networks where people are connected to an event, for instance) in which actors are linked by their common membership of groups consisting of authors of a paper. Co-authorship networks reveal more interaction than many other affiliation networks, and it is probably fair to say that most people who

(C) Juan M.C. Larrosa. Published in Journal of Economics, Finance and Administrative Science. Published by Emerald Publishing Limited. This article is published under the Creative Commons Attribution (CC BY 4.0) licence. Anyone may reproduce, distribute, translate and create derivative works of this article (for both commercial and non-commercial purposes), subject to full attribution to the original publication and authors. The full terms of this licence may be seen at http://creativecommons.org/licenses/by/4.0/legalcode 
have written a paper together are genuinely acquainted with one another (Pepe, 2011). Studies of current literature about co-authorship networks mostly give emphasis to understand patterns of scientific collaborations, to capture collaborative statistics, and to propose valid and reliable measures for identifying prominent author(s). In terms of a broader approach, the network of collaboration developed by an author is part of her social capital (Lin, 2001) that grants endorsement (Ding, 2011) and influence (Brandao and Moro, 2012).

A special call for presenting academic production is a congress. While this institutional form of has been diminishing its importance as a source of scientific knowledge citation (Lisée et al., 2008) each country has developed its own big meeting for each respective branch of the science. So, for Economics for instance, the American Economic Association Annual Meeting in USA and the Encontro Nacional de Economia Política in Brazil are clear examples of congresses that reunite mostly national professionals. In Argentina, there is also a main congress where each year recent graduates submit their production as a way to make public and discuss their results. The common place for exposing new contributions in the Economics profession in Argentina has been the traditional Annual Meeting of the Asociación Argentina de Economía Política (AAEP) where from recently graduated to old school researchers and professors converges yearly for presenting highly diverse contributions (Olivera, 1987). The congress is a first step for recent graduates and the profession in general to submit their contributions and, in many occasions, these become in their first publications in an actual proceeding. It is a signaling event for future $\mathrm{PhD}$ market candidates (Barbezat, 2006). The event represents the gate of entrance to the profession for many of the economists in Argentina. At the same time, contributions also represent the state-of-the-art of the economic knowledge in the country.

We study the role of co-authorship in the academic production submitted to this event. We try to identify patterns in co-authorship, for instance, how co-authorship has evolved? Is there a time pattern in that evolution and what explains it? What universities or research centers have been more prone to develop coauthored contributions? Is there a gender difference between male and female economists? What were the main topics analyzed by these contributions?

The goal of the paper is to present a dimension of the evolution of the economic production and prevalent economic thought in Argentina in a time span of 40 years. We will focus in two main studies: first, the evolution of co-authorship as a way of collaborative endeavor and its influence in the academic production in the fields of Economics in the country, and second, to provide information on the changes that the economic research has shown through the presentations in this congress, as a way of showing how the economic thought has changed in the period. It is valid to remark that most departments of Economics in Argentina participate so the contributions express the main topics and lines of thought and syllabus present in the local academia.

Technically, we gather data, construct networks, and study many local and structural properties of them, among other features such as numbers of papers written by coauthors, the timeline evolution of papers presented, the topics that identify each contribution, and a variety of measures of connectedness within a network. Specifically, we estimate centralities like closeness, eigenvector, Pagerank and betweenness. We also distinguish gender differences in the contributions.

The paper follows with Section 2 where we present introductory concepts and review recent literature. Section 3 presents the event to be studied and analyzes structural metrics. Section 4 presents the network of co-authorship. Section 5 ends the contribution with conclusions. 
JEFAS

24,47

\subsection{Co-authorship networks}

A co-authorship network is depicted by linking authors that participate in a same literary output. As long as other authors collaborate in producing one paper more links are included relating each new coauthor with all other precedent authors. Previous studies consider that co-authorship relation between two authors as a scientific collaboration (Newman, 2001a). It has long been realized that the study of co-authorship of articles in journals provides a window over the patterns of collaboration within the academic community (Newman, 2003). In a detailed analysis of scientific growth as a process of diffusion of knowledge, the most productive or lead authors are often those who introduce new ideas and therefore have a major impact on the scientific community. Several studies have shown that scientific productivity depends, among other things, on scientists' attitude towards collaboration in research.

Co-authorship network, as a relevant type of social network, has been extensively studied (Newman, 2001a, 2001b, 2003). Studies have provided us with a bird-eye view of collaboration patterns in different branches increasing its scientific status as a field of research (Jahn, 2008). There have been few studies on co-authorship networks in Argentina. For instance, Chinchilla-Rodríguez et al. (2012) study a broader approach to Argentinian scientific authors but focusing on publications on indexed journals. Borracci et al. (2009) reveal patterns in scientific collaborations between Argentinian cardiologists analyzing the main academic reviews. The contribution works with a giant component of 571 nodes finding descriptive statistics of the sample, such as average authors per paper, productivity indexes or average papers per author, for instance. Aguado-López et al. (2009) also find patterns of collaboration among coauthors in Argentina in a sample of Latin American journal. Our contribution adds the study of the clusters of relevant topics that depicts main actors and affiliation clusters. In Latin America, Maia et al. (2013) also study an annual academic meeting, specifically a Brazilian symposium on computer networks and distributed systems, but they focus on structural metrics and geographical attributes of the authors. Interestingly, Lisée et al. (2008) study a conference as a source of generation of knowledge in sciences. Most new papers quote a low percentage of papers presented in proceedings, ranging from 1.7 to 20 per cent depending on the specific branch.

We study the way co-authorship between economists who present contributions in a periodical professional meeting in Argentina has been evolved in the past decades. The common place for exposing new contributions in the Economics profession in Argentina has been the traditional Annual Meeting of the Asociación Argentina de Economía Política (AAEP) where from recently graduated to old school researchers and professors converges yearly for presenting a highly diverse supply of contributions (Olivera, 1987).

Important contributions that have added information on network of collaboration between economists are Fafchamps et al. (2006) and Goyal et al. (2006) which present evidence of small world properties in an enormous database of economist contributions. Bukowska et al. (2014) also present a specific study of co-authorship in Polish economists and find that the number of articles written in collaboration and average number of authors per article are steadily increasing, while this collaboration remains small compared to other European countries. These three previous papers focus in structural properties of the network and focus on journal references. The first two conclude, as well as in our case, that the collaboration community of economists is represented by a small world framework, where most contributors belong to a giant connected component and they coexist with several isolated participants (non-connected components).

As shown in Figure 1, the quantity in coauthored contributions in the AAEP Annual Meeting has increased remarkably since the 1990s. Each of the networks depicted in the 


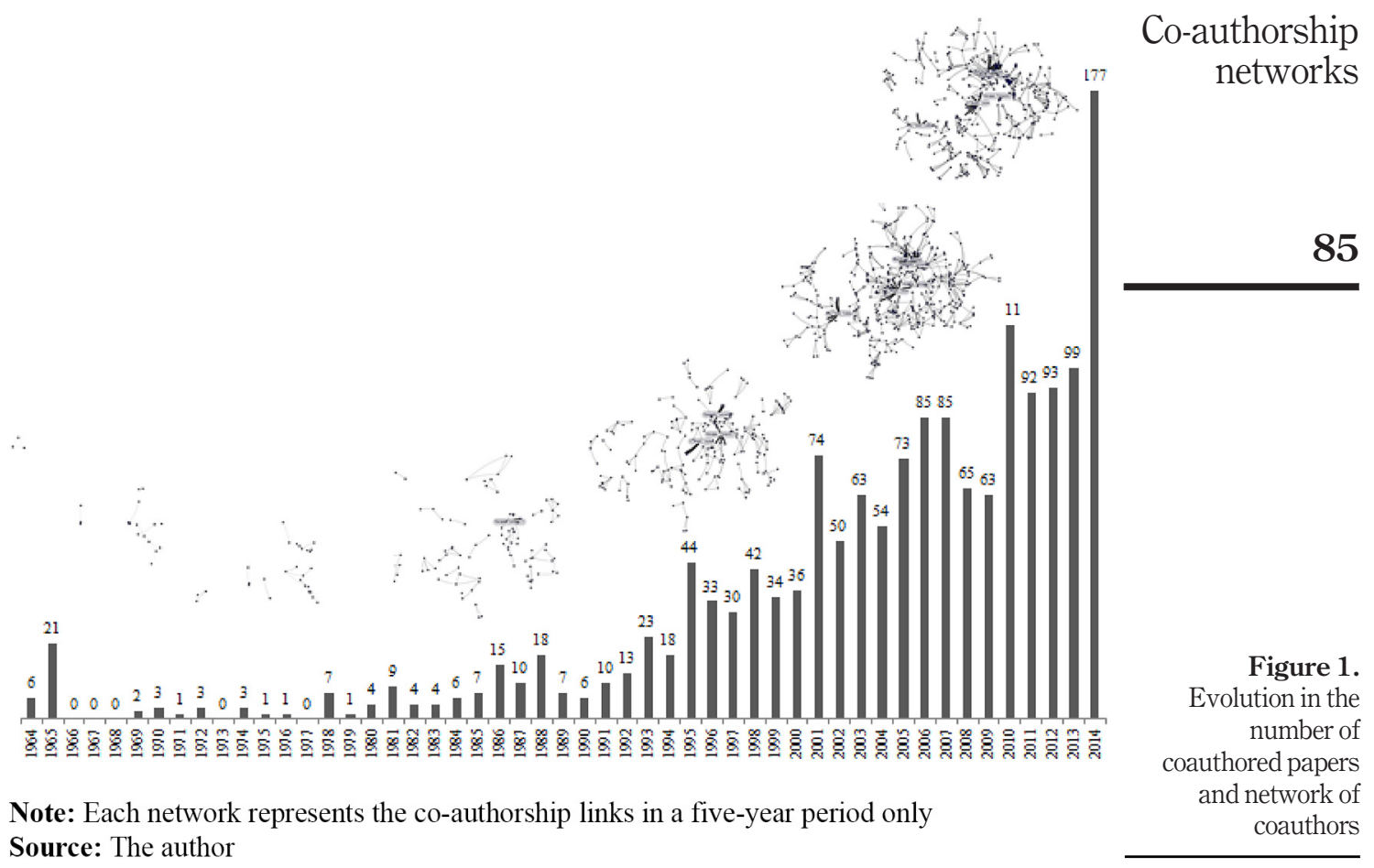

upper position to the bars represents a ten-year window for co-authorship papers. The earliest (network above bars to the extreme left) is for the 1960s, the second for the 1970s and so on. The last network represents the five-year period of 2010-2014. It is clear that networks become larger and more populated. The 1990s marks a notorious increment and the last network also shows that the last five-year period presents a level of activity like previous decade in terms of co-authorship.

We want to explore the pattern of co-authorship networks and to describe the collaboration structure between authors, institutions and topics covered by the contributions. We will make use of metrics of social network analysis, from network level (structural) and to actor level variables. We use clustering (indirect approach), graph metrics (such as density, diameter, etc.), centrality indices; cohesion measures (component, cliques)[1].

\section{Methods}

To undertake this study we identified conference papers published in the AAEP site (www. aaep.org.ar) during 1964-2014. For each paper selected we identified the name and surnames of the authors, as well as their institutional affiliation (institution). The network consists in 890 nodes and 1,644 links. We use NodeXL (Smith et al., 2010) as software packages for data processing and network visualization.

We obtained a series of measures to allow for the analysis of structure or social networks. We analyze agents (authors or institutions) individually, we present three measures of centrality or cohesion that facilitate detailed analysis of the social network studied: degree, and indices of betweenness and eigenvector. Several social network metrics are used that 
JEFAS 24,47

enable to measure the characteristics of a network, including components analysis and centrality analysis.

\subsection{Giant component and centrality measures}

A component of a network is a substructure in which there is at least a path connecting a node and any other node. A network may include some components which are isolated from each other without any connections. The size of a component indicates the number of nodes it contains. Component analysis is used to study the network structure. The main component of a network is called the giant component. All centrality metrics will be performed on the giant component. A component analysis is used to study academic circles of the AAEP proceedings in this paper.

In this study we apply three classic centrality measures (degree centrality, closeness centrality and betweenness centrality) and PageRank (which is a variant of eigenvector centrality) to the co-authorship network.

2.1.1 Degree centrality. Degree centrality equals the number of ties that a vertex has with other vertices. It is a local metric and it does not require the researcher to know the whole network structure. The equation for this measure is as follows, where $d\left(n_{i}\right)$ is the degree of $n_{i}$ :

$$
C_{D}\left(n_{i}\right)=d\left(n_{i}\right)
$$

Generally, vertices with a higher degree or more connections are more central to the structure and tend to have a greater ability to influence others.

2.1.2 Closeness centrality. A more sophisticated centrality measure is closeness (Freeman, 1979). It emphasizes the distance of a vertex to all others in the network by focusing on the geodesic distance from each vertex to all others. Closeness is a metric of how long it will take information to spread from a given vertex to others in the network. Closeness centrality is a global metric and focuses on the extent of influence over the entire network. In the following equation, $C_{C}\left(n_{i}\right)$ is the closeness centrality and $d\left(n_{i}, n_{j}\right)$ is the distance between two vertices in the network:

$$
C_{D}\left(n_{i}\right)=\sum_{i=1}^{N} \frac{1}{d\left(n_{i}, n_{j}\right)}
$$

2.1.3 Betweenness centrality. Betweenness centrality is another global metric based on the number of shortest paths passing through a vertex. Vertices with high betweenness connect different groups. In the following formula, $g_{j i k}$ is all geodesics linking node $j$ and node $k$ which pass through node $i ; g_{j k}$ is the geodesic distance between the vertices of $j$ and $k$ :

$$
C_{B}\left(n_{i}\right)=\sum_{j, k \neq i} \frac{g_{j i k}}{g_{j k}}
$$

For co-authorship networks, vertices with high betweenness connect authors who share similar research interest. Therefore, authors with high betweenness usually engage in research of different fields and thus show interdisciplinarity.

Eigenvector centrality and PageRank. Eigenvector is a global metric that is based on the principle that the importance of a node depends on the importance of its (direct) neighbors. The prestige $x_{i}$ of node $i$ is proportional to the sum of the prestige of the neighboring nodes pointing to it: 


$$
\lambda x_{i}=\sum_{j=j \rightarrow i} x_{j}=\sum_{j} A_{j i} x_{j}=\left(A^{\prime} x\right)_{i}
$$

Co-authorship networks

where $x_{i}$ is the $i$ component of the eigenvector of the transpose of the adjacency matrix with eigenvalue $\lambda$. PageRank is derived from the influence weights formally formulated by the contribution of Page and Brin (1998), who developed a method for assigning a universal rank to webpages based on a weight-propagation algorithm called PageRank. A page has high rank if the sum of the ranks of its backlinks is high. This idea is captured in the PageRank formula as follows:

$$
P R(p)=\frac{(1-d)}{N}+d \sum_{i=1}^{k} \frac{P R\left(p_{i}\right)}{C\left(p_{i}\right)}
$$

where $N$ is the total number of pages on the Web, $d$ is a damping factor, $C\left(p_{i}\right)$ is the outdegree of $p_{i}$, and $p_{i}$ denotes the in-links of $p$. PageRank is, in fact, the principal eigenvector of a transition matrix. PageRank to be a variant of eigenvector centrality, and therefore we classify it as a centrality measure in this study. Actors in the PageRank of Web information retrieval systems are Web pages, and actors in the PageRank of co-authorship networks are authors. If author A coauthors with author B, this is similar to endowing one credit to B; if B has three collaborators, then each of her/his collaborators will have a third of B's credit; the procedure continues in this way until all authors have stable PageRank values. So PageRank does not merely count how many collaborators an author has, but it also considers the impacts of those collaborators.

The earliest pursued category of methodology in the social network analysis is the centrality of individuals and organizations in their social networks. Different kinds of centralities including degree, betweenness, eigenvectror and PageRank, give rough indications of the social power from several different perspectives of a node based on how well they "connect" the network[2]. We will estimate the aforementioned centralities for identifying main actors in the network.

\section{Results}

As mentioned earlier, the AAEP is the Asociación Argentina de Economía Politica (Argentinian Political Economics Association) and congregates most of the professional and academic economists in the country. One annual meeting has been taking place since 1964 in different universities and cities across the country. In 1966 and 1973, the meeting was suspended. As a byproduct of each meeting the association prints the proceedings of each meeting that are actually online in the previously mentioned website of the institution.

Papers have been primarily single-authored with a decreasing trend. As Figure 2 depicts, most papers were single-authored initially up to mid-seventies, but it is with the beginning of the 1990s when single authored papers ceded its preeminence in the proceedings of the congress. By 1999 coauthored contributions surpassed them in quantity. As the ratio Single/ Coauthored $(\mathrm{S} / \mathrm{C})$ shows in the dashed line co-authorship has been steadily increasing $(\mathrm{S} / \mathrm{C}$ ratio decreasing) since the 1970s[3]. That is a result also found in Sutter and Kocher (2004, p. 328): single-authored economic papers descend from 70.3 per cent in 1977 to 46.2 per cent in 1992.

In the graph theory, a connected component of an undirected graph (such as our case) is a subgraph (a network inside the network) in which any two vertices are connected to each other by paths (steps of links connecting nodes), and which is connected to no additional vertices in the supergraph (the rest of the network). The whole network has up to 
JEFAS 24,47

88

Figure 2.

Total papers presented at the AAEP Annual Meeting (singleauthored and coauthored)
17 connected components but the giant component congregates 96 per cent of nodes and 98 per cent of links (Table I). This is a case of the small world phenomenon (SWP) with a big connected component that connects most of the nodes. A very simple measure of SWP is that the natural logarithm of the total of nodes $(\ln (890)=6.74)$ roughly approximates to the average distance among peers (8.45) (Goyal et al., 2006). The diameter of the larger connected component is high requiring as much as 20 steps for beginning with one node to reach its farthest node. The SWP is the observation that one can find a short chain of colleagues, often of no more than a handful of individuals, connecting almost any two authors on the network. It is equivalent to the statement that most pairs of individuals are connected by a short path through the co-authorship network.

Studies confirmed that co-authorship networks follow a power law distribution of node degree with cutoffs (Barabási et al., 2002). A plethora of studies have followed to look into large-scale co-authorship networks to gain a detailed understanding of the power law regime in node degree distribution[4]. For the AAEP annual meeting, estimation is presented in the composite Figure 3. The main figure shows the distribution frequency of the degree among coauthors and the inserted figure represents the same information but in log-log scale. As it is observed, it is a long tail distribution of degrees (fat-tail distribution). This is an indication for the presence of a structure of diffusion of information congregated in hubs, or persons with many connections that reach farther nodes. A network with fat-tail distribution of degree of nodes has good properties for dissemination of information.

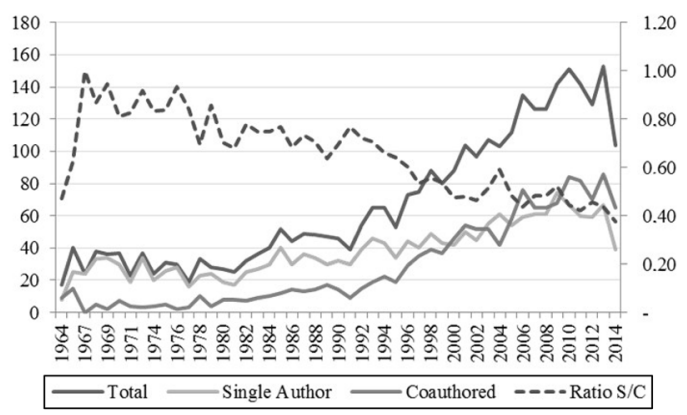

Source: The author

\section{Table I.}

Graph metrics of connected components

\begin{tabular}{lrrrrrrr}
\hline Group & N & UE & EwD & TE & MGD & AGD & D \\
\hline G1 (giant) & 850 & 1442 & 167 & 1609 & 20 & 8.45 & 0.004 \\
G2 & 5 & 10 & 0 & 10 & 1 & 0.8 & 1 \\
G3-G7 & 3 & 3 & 0 & 3 & 1 & 0.667 & 1 \\
G8-17 & 2 & 1 & 0 & 1 & 1 & 0.5 & 1
\end{tabular}

Notes: Codification: $\mathrm{N}=$ Nodes; $\mathrm{UE}=$ Unique edges; $\mathrm{EwD}=$ Edges with duplicates; $\mathrm{TE}=$ Total edges; $\mathrm{MGD}=$ Maximum geodesic distance (diameter); $\mathrm{AGD}=$ Average geodesic distance; $\mathrm{D}=$ Graph density Source: The author 


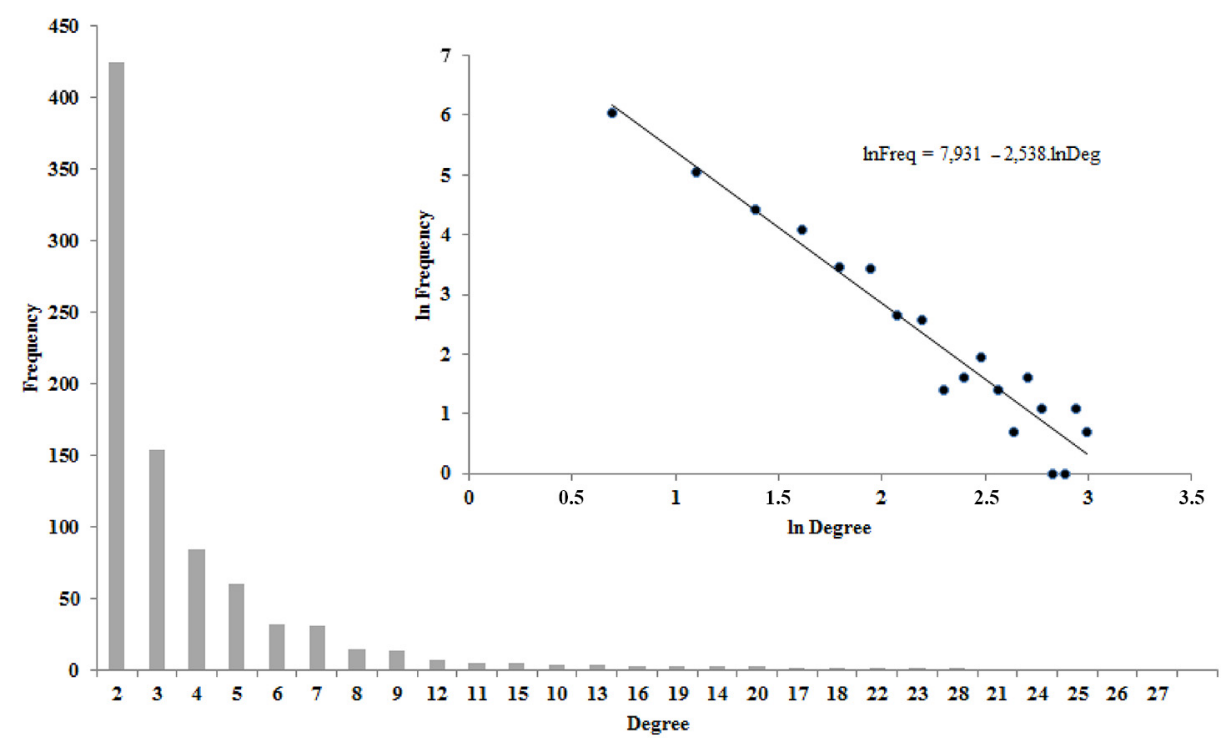

Source: The author

\subsection{Affiliations}

Members of the economic community in Argentina work in diverse public and private institutions. These were classified into 19 national universities (Universidad Nacional de Córdoba-UNC-, Universidad Nacional de La Plata -UNLP-, Universidad Nacional del Sur UNS-, Universidad de Buenos Aires -UBA-, Universidad Nacional de Cuyo -UNCu-, Universidad Nacional de Salta-UNSa-, Universidad Nacional de Rosario -UNR-, Universidad Nacional de Mar del Plata -UNMdP-, Universidad Nacional General Sarmiento -UNGS-, Universidad Nacional del Litoral -UNL-, Universidad Nacional de Río Cuarto -UNRC-, Universidad Nacional de Chilecito -UN Chilecito-, Universidad Nacional de la Patagonia San Juan Bosco -UNPSJB-, Universidad Nacional de San Luis -UNSL-, Universidad nacional de Morón -UNaM-, Universidad Nacional del Centro de la Provincia de Buenos Aires UNCPBA-, Universidad Nacional de Quilmes -UNQ-, Universidad Nacional de Tucumán UNSTA-, Universidad Nacional de San Martín -UNSAM-), 9 private universities (Universidad del CEMA -UCEMA-, Universidad de San Andrés -UDESA-, Universidad Torcuato Di Tella -UTDT-, Universidad Argentina de la Empresa -UADE-, Universidad Católica Argentina -UCA-, Universidad Austral -Uaustral-, Universidad de Belgrano -UB-, Universidad Empresarial Siglo 21 -UES21-, Universidad Católica de Santa Fé -UCSF), several Private/Public Research Center, Foreign Universities, State Entities (ministries, secretaries, and the like), Private/Public Bank, International Entities (UN, CEPAL, among others). Figure 4 shows up the clusters detected by affiliation. Clusters are presented in circular network layout and the thinner links represent intra-group co-authorship and broader links represent extra-group co-authorship. The size of each circle network makes it easy to grasp what affiliations are more prominent than other.

3.1.1 Structural metrics. As Table II shows up the larger affiliation that engenders co-authorship to the meetings is UNC with 130 coauthors followed in sharp difference by the UNLP and UNS, with 42 and 44 coauthors below, respectively.
Co-authorship networks

89

Figure 3. Frequency of degrees and $\log -\log$ representation 
JEFAS

24,47

90

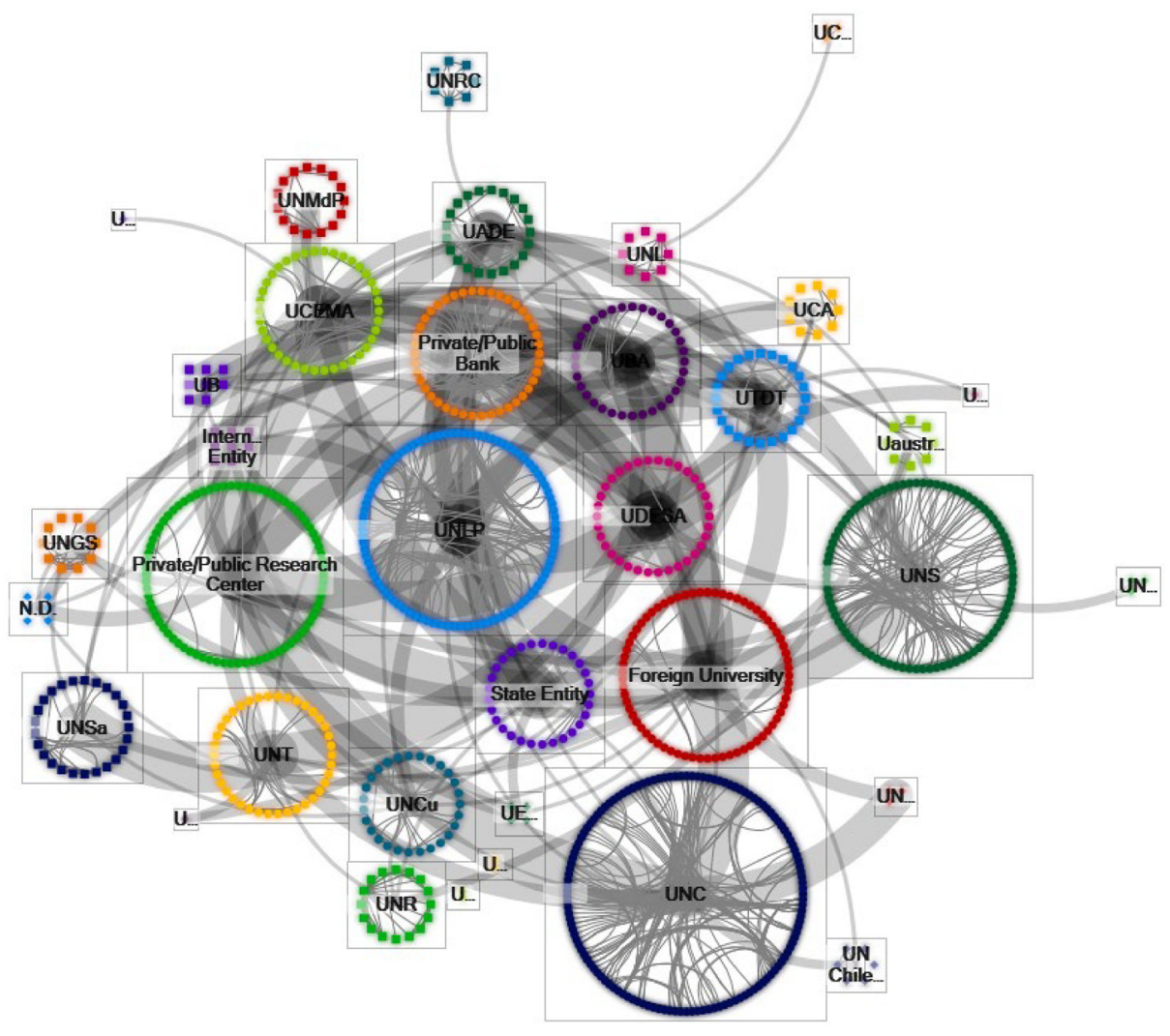

Figure 4.

Cluster by affiliation

Source: The author

Most of co-authorship tends to be done with colleagues or fellows of the same faculty, department or university. A generalization of this result suggests that Rodríguez and Pepe (2008) findings emerges: co-authorship is primarily driven by departmental and institutional affiliation. The role of geography in scientific collaborations is also remarked by the location of each university: academic production tends to focus on regional problems [Guerci Sidone et al. (2014) study the role of geography in Brazilian academic production].

\subsection{Network of co-authors}

When analyzing important actors in the co-authorship sample, we estimate actor's centralities. Gender differences emerge. Figure 5 shows the difference in size in male vs female co-authorship network. Male network is denser with more prominent actors.

A way to notice other gender differences we can anticipate that most centrality calculations sensitively differ when considering male or female co-authorship subnetwork[5]. For instance, Table III shows the deviations from the mean in centralities between genders. Male coauthors surpass (in an almost 3:1 ratio) female colleagues in all centrality measures. Female, on the other hand, shows an upper estimation for clustering coefficient. That would mean women tend to join for writing papers with more common coauthors. This gender gap is also noted in 


\begin{tabular}{lrrrrrrrrrrr}
\hline Label & N & UE & EwD & TE & CC & SVCC & MVCC & MECC & MGD & AGD & D \\
\hline UNC & 130 & 226 & 46 & 272 & 4 & 2 & 125 & 269 & 10 & 4.23 & 0.030 \\
UNLP & 88 & 116 & 17 & 133 & 12 & 9 & 73 & 126 & 8 & 3.54 & 0.032 \\
UNS & 86 & 155 & 6 & 161 & 3 & 2 & 84 & 161 & 8 & 3.85 & 0.043 \\
Foreign University & 63 & 33 & 0 & 33 & 39 & 28 & 7 & 9 & 3 & 1.08 & 0.017 \\
Public/Private Research & 57 & 31 & 0 & 31 & 31 & 20 & 7 & 7 & 4 & 1.22 & 0.019 \\
Central/Private Bank & 41 & 50 & 18 & 68 & 11 & 7 & 27 & 64 & 5 & 2.23 & 0.071 \\
UCEMA & 38 & 38 & 2 & 40 & 12 & 9 & 21 & 31 & 7 & 2.88 & 0.055 \\
UDESA & 34 & 17 & 0 & 17 & 20 & 14 & 8 & 10 & 4 & 1.60 & 0.030 \\
UNT & 34 & 40 & 2 & 42 & 8 & 5 & 25 & 40 & 8 & 3.37 & 0.073 \\
State entity & 29 & 20 & 2 & 22 & 16 & 10 & 5 & 7 & 2 & 0.81 & 0.052 \\
UBA & 29 & 17 & 0 & 17 & 15 & 10 & 7 & 9 & 2 & 1.21 & 0.042 \\
UNCu & 27 & 30 & 0 & 30 & 6 & 4 & 21 & 29 & 8 & 3.29 & 0.085 \\
UTDT & 24 & 13 & 4 & 17 & 12 & 9 & 11 & 15 & 5 & 2.14 & 0.054 \\
UNSa & 22 & 28 & 4 & 32 & 2 & 1 & 21 & 32 & 5 & 2.57 & 0.130
\end{tabular}
Co-authorship
networks

Notes: Codification: $\mathrm{N}=$ Nodes; $\mathrm{UE}=$ Unique edges; $\mathrm{EwD}=$ Edges with duplicates; $\mathrm{TE}=$ Total edges; $\mathrm{CC}=$ Connected components; $\mathrm{SVCC}=$ Single-Vertex connected components; $\mathrm{MVCC}=$ Maximum vertices in a connected component; $\mathrm{MECC}=$ Maximum edges in a connected component; MGD = Maximum geodesic distance (diameter); $\mathrm{AGD}=$ Average geodesic distance; $\mathrm{D}=$ Graph density

Source: The author

Table II.

Cluster metrics of groups with more than 20 nodes

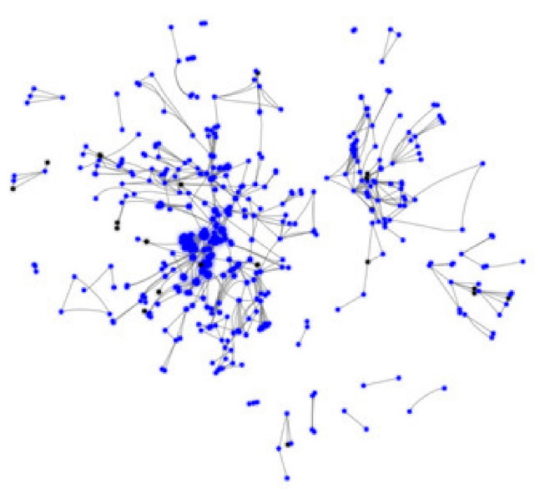

(a)

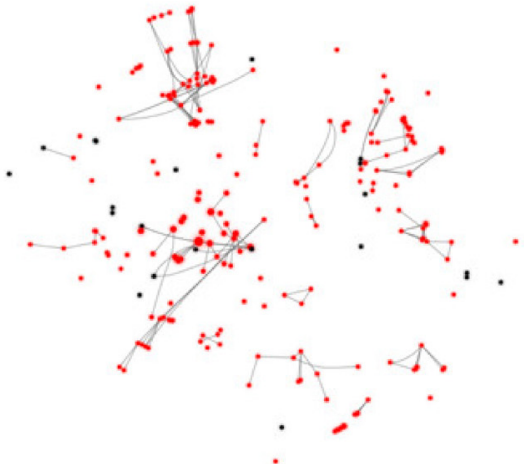

(b)

Notes: (a) Male co-authorship network; (b) female co-authorship network

Source: The author

\begin{tabular}{lcccccc}
\hline & Degree (\%) & $\begin{array}{c}\text { Betweenness } \\
\text { centrality (\%) }\end{array}$ & $\begin{array}{c}\text { Closeness } \\
\text { centrality (\%) }\end{array}$ & $\begin{array}{c}\text { Eigenvector } \\
\text { centrality (\%) }\end{array}$ & $\begin{array}{c}\text { PageRank (\%) } \\
\text { Clustering } \\
\text { coefficient (\%) }\end{array}$ \\
\hline Male & 3.1 & 18.2 & 5.0 & 18.9 & 4.4 & -6.0 \\
Female & -6.4 & -37.0 & -10.9 & -38.6 & -8.9 & 12.3
\end{tabular}

Notes: Sample: Female: 279 individuals; Male: 566 individuals Source: The author

Figure 5.

Male and female coauthorship subnetworks
Table III.

Mean deviations of metric by gender 
JEFAS

24,47

92

Barbezat (2006), where it also points out the presentations in congresses were a significant variable in explaining successful $\mathrm{PhD}$ candidates. Maske et al. (2003) study the determinant of academic production between male and female economists. The work remarks a gender difference in features such as experience and numbers of contributions. Statistically, having experience, the number of previous academic publications and a high level of co-authorship affect positively the productivity of female economists. In any case, a gender gap has been detected across the profession.

Having shown this difference among economists by gender, it is plausible to see more male economists in the top of centrality measures. The following tables will remark this fact. In the top considering the entire sample, given the gender gap presented in Table III, we observe mostly male economists. Table IV shows maxima from male sample (that are also full sample maxima). Actors with the larger betweenness centrality are Fernando Navajas and Santiago Urbiztondo[6] (both from UCEMA). This centrality remarks an economist that tends to connect groups otherwise disconnected and is an indication of interdisciplinarity inside the economic profession. They are both researchers on industrial organization topics with specialization in regulatory economics.

\begin{tabular}{lcclcl}
\hline Author & Degree & Betweenness & Eigenvector & PageRank & Affiliation \\
\hline Gasparini, Leonardo & 28 & 43,564 & 0.04 & 6.289 & UNLP \\
Sosa Escudero, Walter & 23 & 69,798 & 0.041 & 5.221 & UDESA/UNLP \\
Gertel, Héctor & 22 & 21,403 & 0.000116 & 4.689 & UNC \\
Carrera, Jorge & 20 & 39,465 & 0.002 & 5.227 & UNLP \\
Heymann, Daniel & 19 & $85,440^{*}$ & 0.008 & 4.766 & UBA \\
Arrufat, José Luis & 19 & 69,434 & 0.000258 & 4.089 & UNC \\
Porto, Alberto & 19 & 68,620 & 0.019 & 4.783 & UNLP \\
Figueras, Alberto & 18 & 7,219 & 0.000267 & 3.804 & UNC \\
Romero, Carlos & 16 & 31,373 & 0.001 & 3.798 & UADE \\
Díaz Cafferata, Alberto & 16 & 8,243 & 0.000178 & 3.848 & UNC \\
Dabús, Carlos & 16 & 30,281 & 0.003 & 4.004 & UNS \\
Galperín, Carlos & 15 & 21,044 & 0.001 & 4.372 & UBA \\
Sonnet, Fernando H & 15 & 41,244 & 0.000068 & 3.03 & UNC \\
Tohmé, Fernando & 15 & 48,178 & 0.003 & 3.691 & UNS \\
Giuliodori, Roberto & 14 & 3,400 & 0.000075 & 2.887 & UNC \\
Pellegrini, José Luis & 14 & 12,719 & 0 & 2.995 & UNR \\
Navajas, Fernando H & 13 & 114,033 & 0.006 & 3.385 & FIEL \\
Lema, Daniel & 13 & 21,031 & 0.004 & 3.601 & UCEMA \\
Colome, Rinaldo & 13 & 19,706 & 0.00099 & 2.771 & UNC \\
Cicowiez, Martín & 13 & 22,471 & 0.002 & 3.101 & UNLP \\
Ferro, Gustavo & 12 & 17,366 & 0.000163 & 3.24 & UADE \\
Neder, Ángel Enrique & 12 & 18,362 & 0.000087 & 2.764 & UNC \\
del Rey, Eusebio Cleto & 12 & 10,110 & 0.00001 & 3.556 & UNSa \\
Canavese, Alfredo Juan & 12 & 46,950 & 0.013 & 3.084 & UTDT \\
Druck, Pablo & 11 & 7,583 & 0.019 & 2.641 & Fundación del Tucumán \\
Streb, Jorge & 11 & 38,892 & 0.019 & 2.493 & UCEMA \\
Navarrete, José & 11 & 8,400 & 0.000035 & 2.35 & Universitat Jaume I \\
Sanguinetti, Pablo J & 11 & 22,783 & 0.013 & 2.709 & UTDT \\
\end{tabular}

Table IV.

Top male economists sorted by degree
Notes: Only coauthors with degree higher than 10. Figures in italic represent maximum and second highest value for centrality. $\left(^{*}\right)$ Heymann has the second larger betweenness in the table but fourth in the whole male sample

Source: The author 
In the cases of degree and eigenvector centralities, Table IV shows that Leonardo Gasparini (UNLP) has the maximum degree and Walter Sosa Escudero (UNLP and UDESA) has the second maximum. This order is reversed when considering eigenvector centrality, where Sosa Escudero has the maximum followed by Gasparini. Higher degree points to coauthor having worked with more other coauthors and eigenvector points out to coauthor having worked with highly important (in terms of degree) other coauthors. Gasparini is a researcher in income distribution and poverty topics who in fact founded and run a research center, and Sosa Escudero is a well-known econometrician.

PageRank centrality highlights authors that are more solicited by coauthors for producing contributions. Popular authors emerge clearly with this metric, and again, Gasparini (UNLP) and Sosa Escudero (UDESA) jointly with Carrera (UNLP-BCRA) seem to be the most popular coauthors in the event. Carrera is a researcher with specialization in monetary topics.

It is fair to remark that Sosa Escudero is the coauthor that emerges as the only economist in the Top 10 of all centralities as observed in all the previously mentioned tables.

Now let us focus on the gender gap. Table V shows centrality metrics for female coauthor subnetwork. Specifically, it shows the top female economists sorted by at least a degree higher than ten. Heading the list are two BCRA (Argentina's Central Bank) economists: Laura D'Amato and Tamara Burdisso, both specialists in monetary issues.

Table $\mathrm{V}$ shows a different picture in the top ten of the women economist having the highest betweenness centrality metrics. Corina Paz Terán from UNT and Iris Perlbach de Maradona (UNCu)[7] are first and second, respectively. It is interesting to note that more than half of the main female brokers in the network are from universities from the interior of the country. That would mean that women tend to connect groups geographically disconnected. Both economists share common topics of research in labor markets and education.

The results from eigenvector centrality estimation of the Top 10 female economists are also present. This time, emerges Mariana Marchionni (UNLP) and Hildegart Ahumada (UTDT)[8] as the two more important actors. It is worthy to note that Ahumada has less than half that Marchionni's eigenvector centrality and, as noted earlier, Marchionni has only the 65 per cent of the same metric compared to maximum male economist. All of the female economists in the Table V are affiliated to institutions from the Buenos Aires Province. They also both have strong background in econometrics.

Finally, Table $\mathrm{V}$ includes the top ten female economists according to PageRank centrality. This metric is also highly correlated with degree in the female subnetwork, so it is

\begin{tabular}{lccccl}
\hline Author & Degree & Betweenness & Eigenvector & PageRank & Affiliation \\
\hline D'Amato, Laura & 20 & 10,461 & 0.009 & 4.269 & BCRA \\
Burdisso, Tamara & 17 & 17,796 & 0.008 & 3.46 & BCRA \\
Marchionni, Mariana & 15 & 5,953 & 0.027 & 3.293 & UNLP \\
Moscoso, Nebel & 15 & 8,787 & 0.001 & 3.19 & UNS \\
London, Silvia & 12 & 23,854 & 0.001 & 2.921 & UNS \\
Picardi de Sastre, Marta Susana & 12 & 4,881 & 0.000267 & 2.352 & UNS \\
Cerro, Ana María & 12 & 16,327 & 0.000005 & 3.441 & UNT \\
Paz Terán, Corina & 11 & 41,468 & 0.000017 & 2.626 & UNT
\end{tabular}

Notes: Only coauthors with degree higher than 10. Figures in italic character represent maximum and the second highest value for centrality

Source: The author

Co-authorship networks

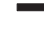


JEFAS

24,47

94

unsurprisingly like Table IV. D'Amato and Burdisso are the most central economists given by this metric, but it is interesting to note that more than half of the top ten are economist from the interior of the country.

Summarizing, men present more important indicators of relevance in terms of co-authorship network than women. It is fair to say that women begin to appear as more numerous in the proceedings by the middle of the 1990s, and from that time on, they are equally or even more represented than men. It seems to be a matter of time that women will fill this gap in the proceedings.

\section{Conclusions}

We have presented data and analysis of co-authorship related to the state of the economics profession in Argentina by analyzing a major event: an annual congress where a vast group of diverse economists in the country regularly exhibits its academic production. In this contribution, we study the impact of certain measures, such as centralities, densities, degree distributions, gender, location and affiliation in the production of economic knowledge. The general structure of the connections promotes diffusion and several actors emerge as hubs of prestige. We study the origin of the papers and presents measures of the importance of different universities and other sources of academic production. Central female and male actors are identified.

We believe that co-authorship it is also a journey to a community not known for any outsider, being from the academia or just any person interested in knowing how people are related in knowledge developing area. Co-authorship network analysis allows any reader to know key players in science branch, main institutions, gender gaps and the like.

\section{Notes}

1. Choobdar et al. (2012) use a motif approach for detecting groups in co-authorship networks.

2. Abbasi et al. (2011) use these metrics for correlating with coauthor personal academic performance.

3. Uddin et al. (2012) also detect an increase in co-authorship during 1990-2009 for a database of Scopus.

4. Milojević (2010) studies specific distributions in co-authorship networks that shown non-power law distribution such as nanotechnologies.

5. Yan and Ding (2009) use centrality measures for estimating the impact of their publication.

6. Santiago Urbiztondo does not appear in the simple given his degree lower than 10.

7. She is not included in the Table $\mathrm{V}$ given that her degree is lower than 10.

8. Also she is not included in the Table $\mathrm{V}$ given that her degree is lower than 10 .

\section{References}

Abbasi, A., Altmann, J. and Hossain, L. (2011), "Identifying the effects of co-authorship networks on the performance of scholars: a correlation and regression analysis of performance measures and social network analysis measures", TEMEP Discussion Paper No. 2011, p. 76.

Aguado-López, E., Rogel-Salazar, R., Garduño-Oropeza, G., Becerril-García, A., Zúñiga-Roca, M.F. and Velázquez-Álvarez, A. (2009), "Patrones de colaboración científica a partir de redes de coautoría”, Convergencia Número Especial IA, pp. 225-258.

Barabási, A.L., Jeong, H., Neda, Z., Ravasz, E., Schubert, A. and Vicsek, T. (2002), "Evolution of the social network of scientific collaborations", Physica A, Vol. 311 Nos 3/4, pp. 590-614. 
Barbezat, D.A. (2006), "Gender differences in research patterns among $\mathrm{PhD}$ economists", The Journal of Economic Education, Vol. 37 No. 3, pp. 359-375, doi: 10.3200/JECE.37.3.359-375.

Borracci, R.A., Doval, H.C., Manente, D. and Tajer, C.D. (2009), "Redes de colaboración científica en las publicaciones cardiológicas argentinas”, Revista Argentina de Cardiología, Vol. 77 No. 6, pp. 487-492.

Brandao, M.A. and Moro, M.M. (2012), "Affiliation influence on recommendation in academic social networks", Proceedings of the AMW.

Bukowska, G., Falkowski, J. and Lopaciuk-Gonczaryk, B. (2014), "Teaming up or writing alone authorship strategies in leading Polish economic journals", Working Papers 2014-29, Faculty of Economic Sciences, University of Warsaw.

Chinchilla-Rodríguez, Z., Ferligoj, A., Miguel, S., Kronegger, L. and de Moya-Anegón, F. (2012), "Blockmodeling of co-authorship networks in library and information science in Argentina: a case study", Scientometrics, Vol. 93 No. 3, pp. 699-717.

Choobdar, S., Ribeiro, P., Bugla, S. and Silva, F. (2012), "Comparison of co-authorship networks across scientific fields using motifs", IEEE/ACM International Conference on Advances in Social Networks Analysis and Mining.

De Stefano, G., Giordano, G. and Vitale, M.P. (2009), Exploring the Pattern of Co-authorship Networks in Different Scientific Domains, Department of Economics and Statistics, University of Salerno, Salerno.

Ding, Y. (2011), "Scientific collaboration and endorsement: network analysis of coauthorship and citation networks", Journal of Informetrics, Vol. 5 No. 1, pp. 187-203, doi: 10.1016/j.joi.2010.10.008.

Fafchamps, M., van der Leij, M. and Goyal, S. (2006), “Scientific networks and co-authorship”, Working Paper No. 256, Department of Economics, University of Oxford.

Freeman, L.C. (1979), "Centrality in social networks: conceptual clarification”, Social Networks, Vol. 1 No. 3, pp. 215-239.

Goyal, S., van der Leij, M.J. and Moraga-González, J.L. (2006), "Economics: an emerging small world”, Journal of Political Economy, Vol. 114 No. 2, pp. 403-412.

Guerci Sidone, O.J., Amaral Haddad, E. and Mena-Chalco, J.P. (2014), "Scholarly publication and collaboration in Brazil: the role of geography", Journal of the Association for Information Science and Technology, Vol. 68 No. 1, pp. 243-258, doi: 10.1002/asi.23635.

Jahn, N. (2008), "The methodological status of co-authorship networks", Fourth International Conference on Webometrics, Informetrics and Scientometrics and Ninth COLLNET Meeting, 28 July - 1 August, Berlin.

Lisée, C., Larivière, V. and Archambault, E. (2008), "Conference proceedings as a source of scientific information: a bibliometric analysis", Journal of the American Society for Information Science and Technology, Vol. 59 No. 11, pp. 1776-1784.

Lin, N. (2001), Social Capital: A Theory of Social Structure and Action, Cambridge University Press, Port Chester, NY.

Maia, G., Vaz de Melo, P.O.S., Guidoni, D.L., Souza, F.S.H., Silva, T.H., Almeida, J.M. and Loureiro, A.A.F. (2013), "On the analysis of the collaboration network of the Brazilian symposium on computer networks and distributed systems", Journal of the Brazilian Computer Society, Vol. 19 No. 3, pp. 361-382.

Maske, L.M., Durden, G.C. and Gaynor, P.E. (2003), "Determinants of scholarly productivity among male and female economists", Economic Inquiry, Vol. 41 No. 4, pp. 555-564, doi: 10.1093/ei/cbg027.

Milojević, S. (2010), "Modes of collaboration in modern science - beyond power laws and preferential attachment", Journal of the American Society for Information Science and Technology, Vol. 64 No. 7, pp. 1410-1423.

Newman, M.E.J. (2001a), "Scientific collaboration networks: I. network construction and fundamental results”, Physical Review E, Vol. 64, pp. 016131.

Co-authorship networks

\section{networks}

95 
JEFAS

24,47

96

Newman, M.E.J. (2001b), "The structure of scientific collaboration networks", Proceedings of the National Academy of Sciences, Vol. 98 No. 2, pp. 404-409.

Newman, M.E.J. (2003), “The structure and function of complex networks”, SIAM Review, Vol. 45 No. 2 , pp. $167-256$.

Olivera, J. (1987), La Asociación Argentina de Economía Politica: Los Años Iniciales, Reunión Anual de la Asociación Argentina de Economía Política, Córdoba.

Page, L. and Brin, S. (1998), "The anatomy of a large-scale hypertextual web search engine", Computer Networks and ISDN Systems, Vol. 30, pp. 107-117.

Rodríguez, M.A. and Pepe, A. (2008), "On the relationship between the structural and socioacademic communities of a coauthorship network", Journal of Informetrics, Vol. 2 No. 3, pp. 195-201, available at: https://doi.org/10.1016/j.joi.2008.04.002

Smith, M. Milic-Frayling, N. Shneiderman, B. Mendes, Rodrigues, E. Leskovec, J. and Dunne, C. (2010), "NodeXL: a free and open network overview, discovery and exploration add-in for excel 2007/2010", available at: http://nodexl.codeplex.com/ from the Social Media Research Foundation, www. smrfoundation.org

Sutter, M. and Kocher, M. (2004), "Patterns of co-authorship among economics departments in the USA", Applied Economics, Vol. 36 No. 4, pp. 327-333, doi: 10.1080/00036840410001674259.

Uddin, S., Hossain, L., Abbasi, A. and Rasmussen, K. (2012), "Trend and efficiency analysis of co-authorship network", Scientometrics, Vol. 90 No. 2, pp. 687-699.

Yan, E. and Ding, Y. (2009), "Applying centrality measures to impact analysis: a coauthorship network analysis", Journal of the American Society for Information Science and Technology, Vol. 60 No. 10, pp. 2107-2118, doi: 10.1002/asi.21128.

\section{Corresponding author}

Juan M.C. Larrosa can be contacted at: jlarrosa@uns.edu.ar

For instructions on how to order reprints of this article, please visit our website: 